\title{
Inadvertent isolation of a focal tachycardia within the superior vena cava
}

\author{
Milko K. Stoyanov, Tchavdar N. Shalganov*
}

Cardiology Department, National Heart Hospital, Sofia, Bulgaria

Email: *tchavdar.shalganov@gmail.com

Received 13 August 2012; revised 20 September 2012; accepted 31 September 2012

\begin{abstract}
The superior vena cava (SVC) is known to be a potential source of focal atrial tachycardias. Not uncommonly these tachycardias trigger atrial fibrillation or flutter. Focal ablation is safe and effective in eliminating arrhythmogenic foci within the SVC. We present the case of a patient with focal atrial tachycardia arising from the SVC. During presumably focal ablation inadvertent electrical isolation of the SVC from the right atrium was achieved, with restoration of sinus rhythm in the atria and persistence of the tachycardia within the SVC.
\end{abstract}

Keywords: Atrial Tachycardia; Superior Vena Cava; Mapping; Electrical Isolation; Catheter Ablation

\section{INTRODUCTION}

Sources of atrial tachycardia can be located at different sites in both atria. Some ectopic foci in the superior and the inferior caval veins can trigger arrhythmias like atrial fibrillation and atrial flutter. Radiofrequency (RF) catheter ablation is safe and highly effective in eliminating such electrical foci, thus preventing recurrences of atrial fibrillation and atrial flutter [1-4]. The usual approach is to target directly the focus of the tachycardia.

\section{CASE REPORT}

The case is presented of a 60-year-old male patient with recurrent sustained atrial tachycardia and atrial fibrillation. Previous treatment with propafenone and verapamil proved to be ineffective. His previous medical history included arterial hypertension, mild degenerative aortic stenosis, and polycythemia vera. The 12-lead electrocardiogram (ECG) during atrial tachycardia showed narrow QRS complex, and atrioventricular conduction ratio varying from $1: 1$ to $3: 1$. The $\mathrm{P}$ wave polarity was similar to that in sinus rhythm-negative in leads aVR and V1, isoelectric in lead aVL, and positive in all remaining

*Corresponding author. leads. The $\mathrm{P}$ wave in sinus rhythm was biphasic with initial positive deflection in lead V1. Noteworthy, the P wave in all leads was narrow-based during tachycardia (Figure 1A). Electrophysiological study was done using 20-polar halo catheter deployed in the right atrium around the tricuspid annulus, 4-polar catheters over the His bundle area and in the right ventricle, and 6-polar catheter in the coronary sinus. Mapping and ablation were performed using 4-mm-tipped RF ablation catheter. The clinical tachycardia was reproducibly induced with incremental and programmed atrial pacing. The earliest atrial activation during tachycardia (50 ms ahead of the $\mathrm{P}$ wave on the surface ECG) was found to be in the superior vena cava (SVC) close to the anteroseptal part of the atriocaval junction. Entrainment from the cavo-tricuspid isthmus gave very long post-pacing interval. Entrainment within the SVC did not alter the activation sequence and demonstrated post-pacing interval only 9 milliseconds longer than the tachycardia cycle length (Figure 1B). RF energy in temperature-controlled mode at a power of 25 Watts and maximum temperature of $52^{\circ} \mathrm{C}$ was delivered within the SVC targeting the earliest discrete local potential preceding the earliest atrial potential, recorded by the halo-catheter. After $14 \mathrm{RF}$ applications caval-atrial block 2:1 ensued, so as the atrial rate decreased, but the tachycardia did not stop (Figure 2A). The next 4 RF applications resulted in restoration of sinus rhythm in both atria, yet the tachycardia was still ongoing in the SVC (Figure 2B), thus showing electrical disconnection of the SVC from the right atrium. Two more RF applications terminated the tachycardia itself. Reversal of the activation sequence ensued, with the SVC potential appearing now late after the right atrial potential in a 1:1 fashion (Figure 3A). Pacing the proximal coronary sinus showed 2:1 atrial-caval block (Figure 3A). Two more RF applications lead to disappearance of the SVC potential (Figure 3B). After the ablation neither atrial tachycardia nor atrial fibrillation could be induced by rapid burst and programmed atrial stimulation with up to three extrastimuli even during hexoprenaline infusion. Six months later the patient was arrhythmia free off antiarrhythmic drugs. 

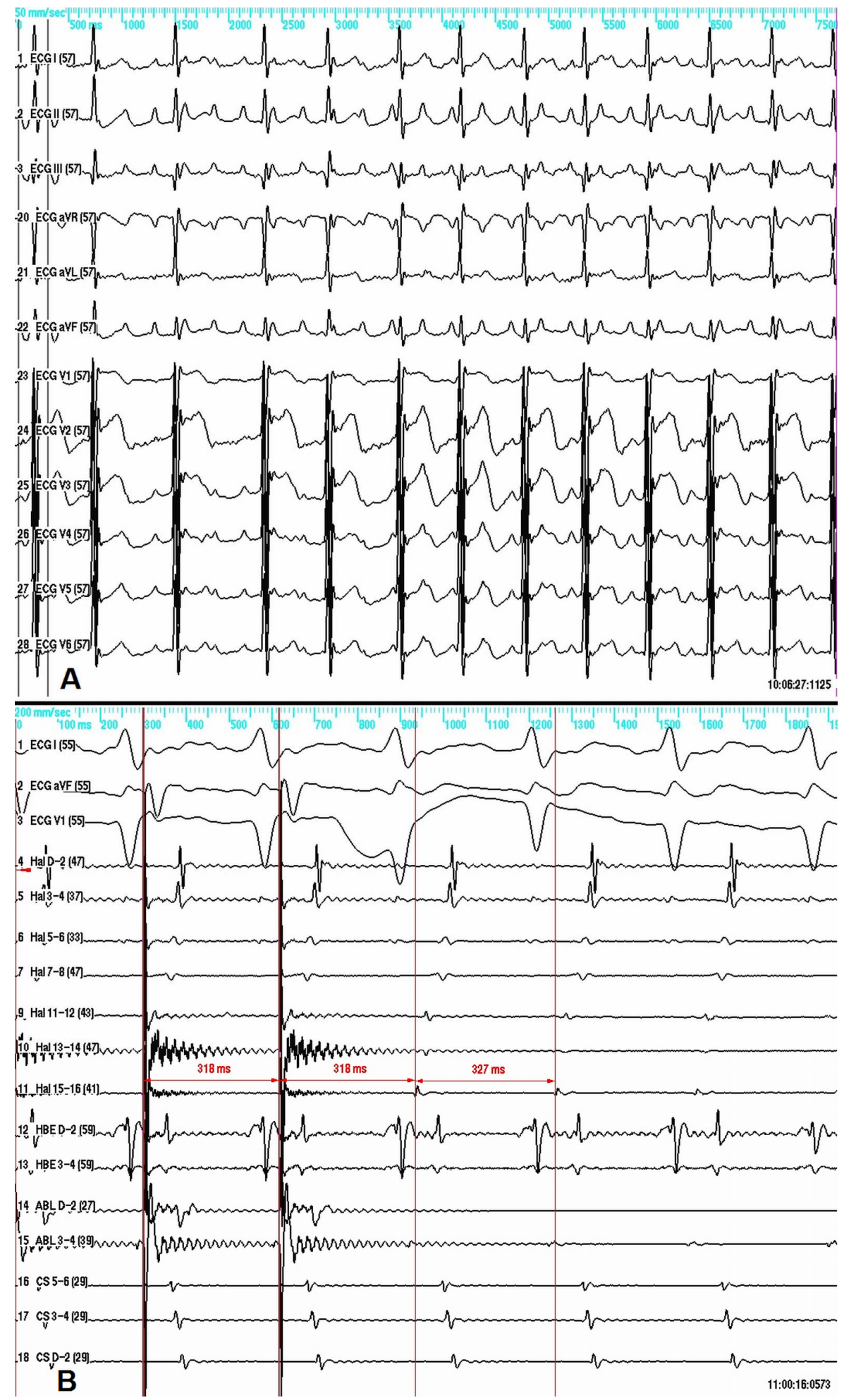

Figure 1. A-12-lead ECG of the clinical tachycardia. B-entrainment within the SVC at a pacing cycle length of $318 \mathrm{~ms}$ results in unaltered activation sequence and short post-pacing interval of 327 ms measured at the proximal electrode pair of the ablation/pacing catheter (Abl 3-4). From top to bottom shown are ECG surface leads I, aVF, V1, and intracardiac electrograms. Hal-multipolar catheter around the tricuspid annulus; HBE-His bundle; Abl-Ablation catheter; CS - Coronary sinus. Abbreviations are the same on all other figures. 


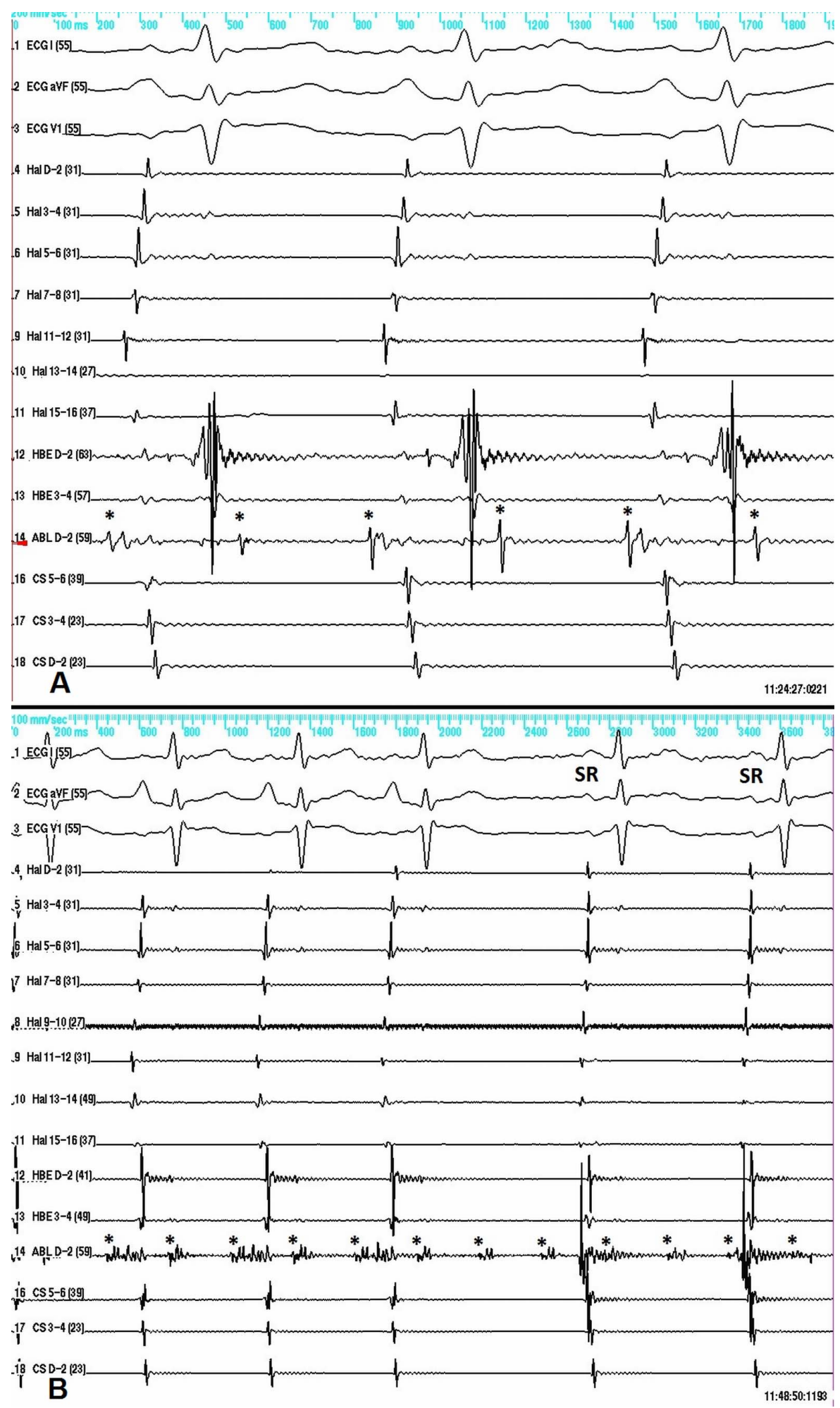

Figure 2. A—caval-atrial block 2:1 during tachycardia. The ablation catheter records a discrete SVC potential (asterisk) at a rate of 204 beats per minute. B-electrical isolation of the SVC demonstrated by resumption of sinus rhythm in the atria, while the tachycardia in the SVC is still running at a cycle length of 294 ms. The former RF applications have transformed the initially sharp and narrow SVC potential into fragmented lower amplitude potential. SR-Sinus rhythm P-wave. Here and on the next figures the SVC potential is marked by an asterisk. 


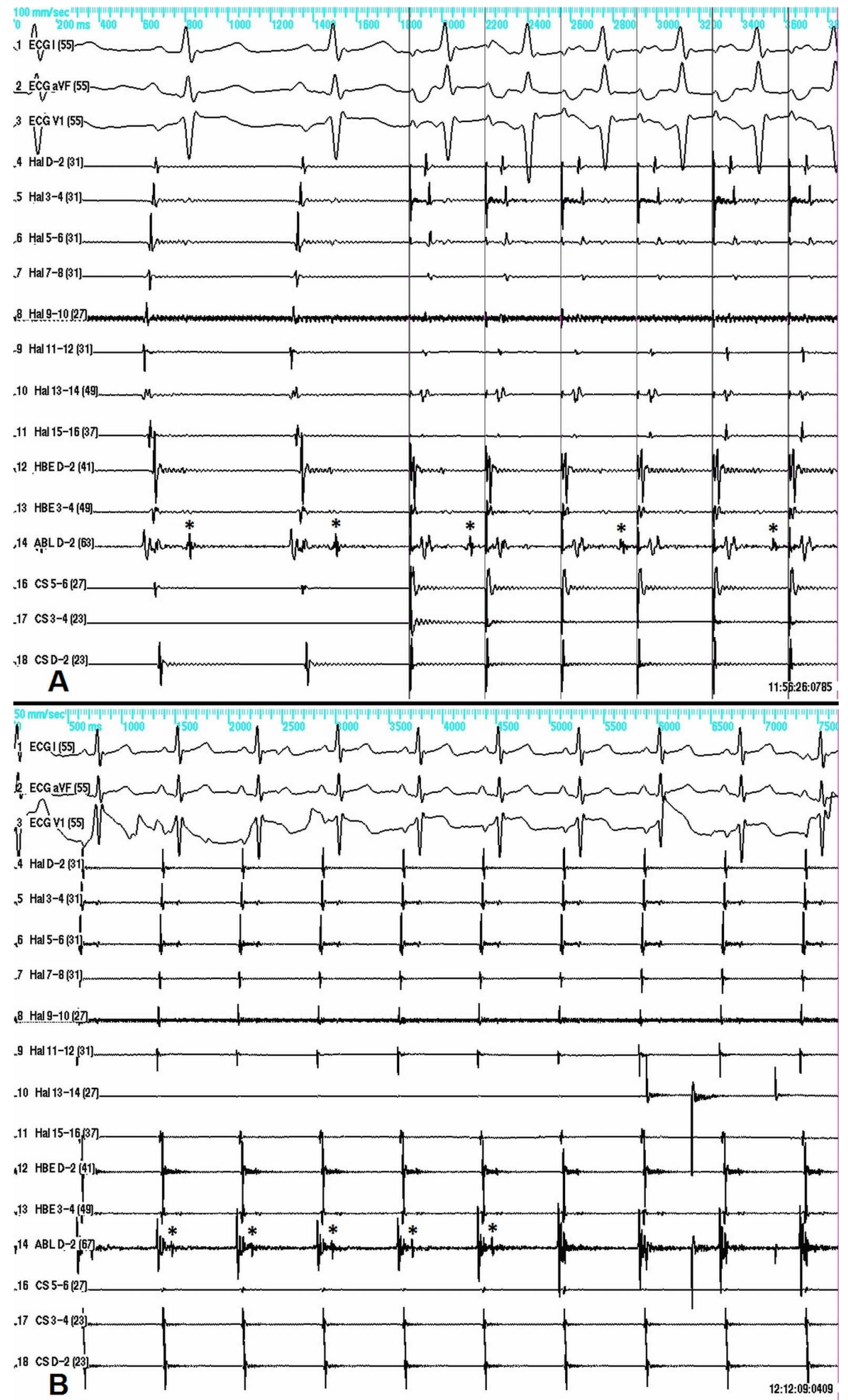

Figure 3. A - slow conduction between the right atrium and the SVC in sinus rhythm (left side of panel A), and atrial-caval block 2:1 during atrial pacing (right side of panel A). B-sudden disappearance of the SVC potential (asterisk) recorded by the ablation catheter during RF energy delivery. 


\section{DISCUSSION}

The origin of focal atrial tachycardia within the SVC is associated with the presence of myocardial sleeves in the atriocaval junction and up in the SVC $[1,2,5,6]$. These myocardial extensions may spread up to $44 \mathrm{~mm}$ in the SVC and be the source of high frequency electrical depolarizations causing atrial tachycardia and/or atrial fibrillation $[1,2,4]$.

In our case as in others [1] the ECG was suggestive of an origin in the upper part of the right atrium that was later confirmed by the intracardiac activation sequence. The narrow-based $\mathrm{P}$ wave is compatible with septal location of the originating focus, where the activation front excites both atria simultaneously and therefore more rapidly. In our case the breakthrough from the SVC to the right atrium was located anteroseptally which explains the ECG characteristics of the P wave.

In contrast to previous publications the tachycardia cycle length in our case was rather regular, varied within narrower limits and did not exceed an upper heart rate of approximately $200 \mathrm{bpm}$. Interestingly, in previous reports pacing maneuvers were either not performed or not successful at inducing or elucidating the arrhythmia mechanism $[1,2,4]$. We were able to show reproducible induction of the clinical tachycardia by pacing that reliably excludes automatic mechanism. Entrainment pacing response is in favor of reentrant mechanism [7].

Atrial tachycardia can be linked to other paroxysmal arrhythmias that can be prevented by RF ablation of the focus of tachycardia $[1,2,4]$. Isolation of the focal source is also possible as shown in our case. This made possible the temporary coexistence of two heart rhythms at the same time- the normal sinus rhythm and the atrial tachycardia. Irrespective of the successful restoration of sinus rhythm and subsequent termination of the tachycardia within the SVC we were not able initially to completely dissociate the SVC potential from the right atrial electrical activity. Hence, we persisted and were able to achieve complete atrial-caval block as shown by SVC potential elimination. Our findings further confirm the existence of a conduction pathway between the essentially extracardiac site of origin of the tachycardia and the right atrium.

\section{ACKNOWLEDGEMENTS}

The authors thank Actavis Bulgaria for covering the article-processing charges for this publication.

\section{REFERENCES}

[1] Chang, K.C., Lin, Y.C., Chen, J.Y., Chou, H.T. and Hung, J.S. (2001) Electrophysiological characteristics and radiofrequency ablation of focal atrial tachycardia originating from the superior vena cava. Japanese Circulation Journal, 65, 1034-1040. doi:10.1253/jcj.65.1034

[2] Tsai, C.F., Tai, C.T., Hsieh, M.H., Lin, W.S., Yu, W.C., Ueng, K.C., et al. (2000) Initiation of atrial fibrillation by ectopic beats originating from the superior vena cava: electrophysiological characteristics and results of radiofrequency ablation. Circulation, 102, 67-74. doi:10.1161/01.CIR.102.1.67

[3] Katsivas, A.G., Manolis, A.G., Vassilopoulos, Ch., Ioanidis, P., Giotopoulou, A. and Kyriakides, Z. (2004) Electroanatomical mapping of a right atrial tachycardia originating within the inferior vena cava. Hellenic Journal of Cardiology, 45, 187-190.

http://www.hellenicjcardiol.org/archive/full_text/2004/3/ 2004_3_187.pdf

[4] Liu, T.Y., Tai, C.T., Lee, P.C., Hsieh, M.H., Higa, S., Ding, Y.A., et al. (2003) Novel concept of atrial tachyarrhythmias originating from the superior vena cava: Insight from noncontact mapping. Journal of Cardiovascular Electrophysiology, 14, 533-539. doi:10.1046/j.1540-8167.2003.02473.x

[5] Nathan, H. and Eliakim M. (1966) The junction between the left atrium and the pulmonary veins: An anatomic study of human hearts. Circulation, 34, 412-422. doi:10.1161/01.CIR.34.3.412

[6] Ho, S.Y., Anderson, R.H. and Sánchez-Quintana, D. (2002) Gross structure of the atriums: More than an anatomic curiosity? Pacing and Clinical Electrophysiology, 25, 342-350. doi:10.1046/j.1460-9592.2002.00342.X

[7] Chen, S.A., Chiang, C.E., Yang, C.J., Cheng, C.C., Wu, T.J., Wang, S.P., et al. (1994) Sustained atrial tachycardia in adult patients: Electrophysiological characteristics, pharmacological response, possible mechanisms, and effects of radiofrequency ablation. Circulation, 90, 1262 1278. doi:10.1161/01.CIR.90.3.1262 